\title{
The Self-Fulfilling Discriminations and Mismatches for Old Workers and Poor Workers
}

\author{
Weiguang Liu \\ Correspondence: Weiguang Liu, Graduate School of Economics, Nagoya University, Aichi, Japan
}

Received: August 21, 2017

Accepted: December 4, 2017 Available online: December 18, 2017

doi:10.11114/aef.v5i1.2606

URL: https://doi.org/10.11114/aef.v5i1.2606

\begin{abstract}
There exists very general discriminations and mismatches for the old workers and poor workers. This paper built a model to explain the reason of that phenomenon based on asymmetry information. A skilled worker may be mismatched with unimportant jobs because of the social bias towards old workers and poor workers, in that situation, the expense workers spend to become skilled will not be covered, which will be a big disincentive for workers to be skilled. The disincentive will lead to less skilled workers, so the working performance of certain kinds of workers in the whole society will be worse, which will confirm the discrimination. In that process, the expense to be skilled is a key factor. And we also try to use that model to explain some phenomena, such as East Asian miracle and class solidification.
\end{abstract}

Keywords: asymmetry information, self-fulfilling discrimination, mismatch, Confucianism, self-esteem, patience, class solidification

\section{Introducation}

Age discrimination and class discrimination are general stereotype discriminations all around the world (Shore and Goldberg, 2005; Capuano, 2016). And those two discriminations usually intertwine with each other (Bastos et al., 2014). In many industrialized countries, population is aging rapidly (McDaniel et al., 2015) and the gap between rich and poor is growing (Saunders et al., 2016). So we need to pay attention to those two discriminations more than ever.

Aging is a process that will deteriorate working performance in most jobs (Antonenko and Flöel, 2014). And being poor will also affect the workers ability to become skilled (Becker and Tomes, 1979; Solon, 2004; Blumkin and Sadka, 2005; Hassler et al., 2007a). So, some degree of discrimination against old workers and poor workers cannot be regarded as irrational, especially when the exact information of each worker is costly and difficult to get (Gandy et al., 2016).

There are many paper discussing the effects of those discriminations (Kunze et al., 2011), but as far as I know, researches analyzing the cause of discrimination are relatively fewer (Becker, 2010). In this paper, we introduce the definition of discrimination as a rational choice under asymmetry information. Firm agents try to maximize their expected profits by treating different types of working force when it is difficult or costly to get the exact information of every worker.

The stereotype discrimination for the old workers and poor workers also has a risk of become self-fulfilling (Glover et al., 2017). With asymmetry information, the Gresham's Law will function to make matters worse (Akerlof, 1970). Gresham's Law can also be explained as 'Bad money drives out good'. In the work force, that means, in a discriminated group, a skilled and qualified worker will not be treated fairly. For example, there may exist a high probability that he will be regarded as unskilled. That would be a huge disincentive for workers in this group to become skilled. As a result, the proportion of unskilled workers in that group will grow. That will lead to a lower average skill of that group. Finally, the discrimination for this group will be reinforce. Just as shown in Figure 1. Here we use old workers as the example. In fact, it also applies to other kinds of vulnerable groups of workers, such as poor workers and wokers of some races.

The key problem is, skilled workers and rich workers are not always the same workers. Skill and high wage have a positive relation but that is not a one-to-one match. Mismatch is a common thing in the labor market (Leuven et al., 2011). One of the purpose of this paper is to explain why this mismatch will exist and discuss the position of equilibrium by considering relative factors. We begin from skill of workers and then we will discuss wage part. 


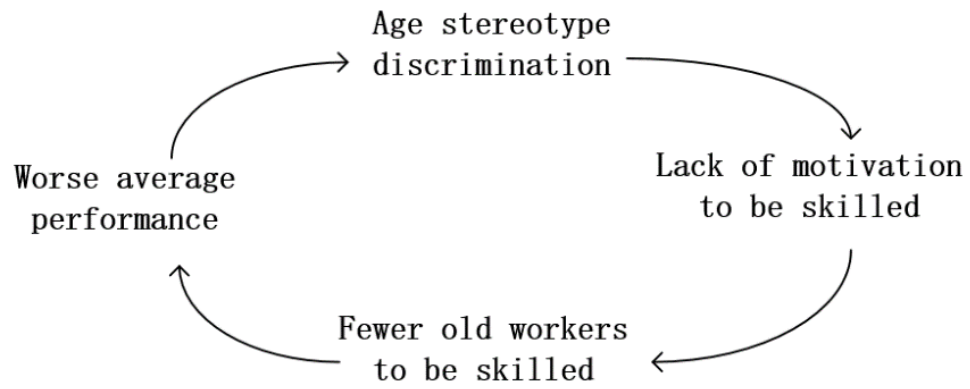

Figure 1. The self-fulfilling mechanism for old workers

Source: Drawn by the author

There are a lot of researches try to find the factors that determines the proportion of skill workers in the whole workforce. Some try to build self-fulfilling mechanisms to solve that problem (Hassler et al., 2007a; Arawatari and Ono, 2009, 2013). And the key in a self-fulfilling mechanism is usually expectation of agents. That is, people's expectation of the future will change the future. Most of those studies are interesting and thought-provoking. Those researches focus on the impact of redistribution policy and are convincible when they talk about welfare states like some North European countries and compare those countries to other developed countries with less welfare (Arawatari and Ono, 2015). But when we focus on developed countries with less welfare, like America, and try to find the factors that determine the proportion of skilled workers, redistribution policy may not be the single most important factor. So, we tried to solve that problem by focus on discrimination and mismatch in labor market.

In this paper, we followed the idea in the model of Arawatari and Ono (2015). That is, workers have a second chance to choose whether to be skilled or not. Examples of educational investment in the later stage of life include part-time study at university, job-training programs, and so on (Arawatari and Ono, 2015).

In addition, there are several questions can be solved by our model in through a new and interesting approach although some questions have been discussed by previous studies. First, what is the economic reason that some workers choose not to be skilled when it is economically available and seems profitable? Second, what is the economic reason of the personalization in education and training? Third, what determines the evolution of a certain type of workers' performance? Fourth, what determines whether the discrimination for certain type of workers will deteriorate? Fifth, what is the relationship between the Confucianism and East Asian economic miracle? Finally, why in most developed countries, after the second world war, experienced a decline of social mobility?

Those questions need further discussion in an improved model. Based on the existing self-fulfilling models, we try to make some reasonable improvements.

First, we loosened the financial constrains and focused on workers' trade-off between current consumption and expected returns in the future. Some of previous models are based on the constraint of workers income and trey assume no loan to support poor workers to finish their education and training (Arawatari and Ono, 2015). Those assumptions are true in many developing countries. However, in most industrialized countries, student loans and other loans are very prevailing (Avery and Turner, 2012), which means workers choose to be skilled or not for some other reasons. For example, in many European countries, as the social welfare and education system is getting better and better, many young people choose to go to go to college or not and choose how much education to get not based on economy constraint, but based on their own expectations of future and the discount factor. Getting higher education is usually possible but not always profitable. So, our model need to make plausible explanations to that.

Second, the introduction of asymmetry information. In the previous studies on this subject, the cost of information is usually ignored, and every agent is deemed as omniscient like god. Such assumptions will certainly make it hard to explain problems like job mismatches in the labor market, and why someone refuse to become skilled even if being skilled seems profitable at first sight. In this paper, on firms' side, we make a clear distinction between overall information and information of individual workers, the cost to get former is negligible, but the cost of latter is usually too high. On workers' side, we try to explain the workers choice to be skilled or not by asymmetry information and discrimination. That is, workers have expectations of the future and they know even if they are skilled, they may not be treated fairly.

Third, we put an emphasis to individual inherent differences and make more delicate divisions, based on individual gifts and on individual costs. Former studies usually assume all workers are with the same intelligence. And given other things, such as wealth status equal, the costs they will need to pay to become skilled are the same (Arawatari and Ono, 2015). In the real world, however, some intelligent students can get scholarship to cover their school expenses and they 
need less effort to become skilled workers. Some others do not have that advantage and need to pay more money and effort to become skilled. That phenomenon will certainly affect the choices of students and workers. Some papers make division like 'high ability group' and 'low ability group', but workers in each group are still identical (Hassler et al., 2007b). In this paper, we take a step further by introducing a continuous distribution of expense a worker need to become skilled, which correspondent with their intelligence.

Fourth, we introduced a new situation where former studies had hardly reached. That is, if a worker was trusted with an important job but he fails, what will happen to him and to the firm? This situation is correspondent with asymmetry information. That is, firms can make mistakes because they use overall information to judge workers in the same group. That situation usually happens in the really world but is hardly touched by previous self-fulfilling mechanisms. This situation is important because it can determine both firms' and workers' choices. In that situation, we take the externality into consideration. This paper assumes a worker's failure will affect other workers' performance, and this loss will be higher if the failed old worker has been given more trust. For the failed worker, he will suffer humiliation, which can be regarded as a negative utility. Like the mechanism demonstrated in Max Weber's Protestant Ethic and the Spirit of Capitalism (Weber, 2002), Confucianism stresses the traditional boundaries of ethical responsibility (Park and Chesla, 2007), which will make that humiliation effect stronger in East Asian countries. We will show how that will relate to East Asian miracle (Liang, 2010) in a new way.

In a word, this paper combines several different fields of studies, such as asymmetry information, adverse selection, externality, opportunity cost, time cost, Cobweb Theorem, intergenerational mobility, behavioral economics and cultural economics. We also brought some ideas from psychology into our paper, such as self-esteem (Maslow, 1943). With all those efforts, we hope this paper can provide more plausible explanations about the workers' performance and wages and provide some inspiration to latter researchers.

The organization of this paper is as follows. Section 2 explains how the self-fulfilling mechanism functions in each group. Section 3 provides the characterization of partial equilibrium and examines the effects of some key factors. Section 4 provides the general analysis and makes some discussion on subjects like class solidification. Section 5 provides concluding remarks.

\section{The Model}

Consider a discrete time economy where time is denoted by $\mathrm{t}=0,1,2, \ldots$. The economy is made up of overlapping generations of individuals. In each period t, first, the firm act according to the general skill level of workers in current period $t$. Then, the workers can observe the overall attitude of the firm and make their decisions about their education and training in the current period $t$, considering their future expected utilities in period $t+1$.

Before moving on, I would like to make clear about the usages of some important concepts in the paper. Some concepts will represent specific meanings to explain specific situations. If the reader feels lost when reading the latter part of the paper, maybe he can look up for the definitions of the concepts in that part.

a. Important task. In the paper, the word 'important task' refers to the tasks with some difficulty and the gain from completing it is high. It can be completed if and only if this individual is skilled. This task cannot be completed by an unskilled old worker, however. And these tasks will involve cooperation with other workers. In those task, one worker's failure will do harm to others' work. That is, there exist negative externality. The more a worker is trusted, the more others will cooperate with them, and the higher that negative externality will be if he fails, an extreme example is Barring Bank collapsed due to one of its head derivatives trader's mistake (Greener, 2006). Important task is usually associated with honor and the worker will feel humiliated if he fails an important task.

b. Expense to be skilled. In the paper, an old worker can become skilled at the expense of his time, money and so on. Here I must make it clear that this expense not only includes financial expense but also non-financial expense such as more intention paid to the work or less time with their family. These expenses are sunk cost and can't be taken back. This kind of expenses are different among workers. Some workers need very low expense to become skilled because of inherent good gifts and good physical and mental health. Some need very high high expense to become skilled because of inherent poor gifts or poor physical and mental health. Most old workers are continuously allocated between the two extremes.

c. Discrimination. The word 'discrimination' in economics is an economic concept instead of a moral concept, a group concept instead an individual concept. In economy, discrimination means distinguishing. And that is an attitude to a whole group of workers. In a simpler word, the firm will treat each group of workers differently. In this paper, discrimination means regarding the old workers group and poor workers group as unskilled groups and to allocate fewer important tasks to them.

d. Trust. To avoid confusion, we sue 'trust' instead of 'discrimination' here to indicate the firm's attitude to a single worker. If a worker is trusted, important tasks will be allocated to him. If a group of workers are discriminated, less 
workers in that group will be trusted with important tasks.

e. High wages. As this paper focus on the changes in comparison and I assume all worker will get their basic wages paid for unimportant tasks no matter he is skilled or not. So, the basic wage is out of the discussion. The wages discussed in this paper refers to bonus paid for completing difficult tasks which need the workers to be skilled. In the paper, the high wage is normalized to 1 .

f. The critical expense. Being skilled involves an expense. In that paper, the expense is sunk cost. If the expense makes a worker indifferent between being skilled or not, that expense is the critical expense. A worker with critical expense is an indifferent old worker in his group.

g. Average skill. Average skill is not real skill of a worker but is an analysis tool in this paper. A worker can only choose to be skilled or not. If he is skilled, his skill degree is 1 . If he is unskilled, his skill degree is 0 . The proportion of skilled workers is also the average of their skill degree. The firm refer to that when making decisions.

h. Degree of trust. Workers in different age groups and wealth classes will be trusted in different degrees. That is, some workers will be regarded as more possible to be skilled while others will be regarded as more likely to be unskilled. Here we use the proportion of trusted workers in a certain group to represent that degree of trust.

i. Magnitude of extra loss. When workers fail important tasks, the firm will not get the income. What's more, the firm will also suffer an extra loss. That loss is caused by failure in cooperation with co-workers. The more a worker is trusted, the more other workers will be willing to cooperate with him, and the more the loss will be if he fails.

In a word, the main source of the problem is that the firm and of the worker are very different. For a worker, he can be in one of the two different conditions: skilled or not. And he has full knowledge of which condition he is, because that is his choice. That is, 'pay the expense' is totally corresponding to skilled and 'not pay the expense' is totally corresponding to unskilled. However, for the firms, it is too costly to get full information of every worker, so the firms tend to judge a worker's skill by the average skill of some group this worker belongs to.

In the previous studies, researchers usually assume the workers' expenses to be skilled is a fixed value and there is a probability of succeed to become skilled. that is plausible in the past several decades. Because in the past, standardization in education and training is very pervasive in industrialized countries. However, recently, personalization of education and training becomes an obvious tendency all around the world (Pykett, 2009). Workers can choose education and training more flexible, both in quantity and quality. High ability workers can become skilled with less expense. Low ability workers can choose between paying high expense to be skilled or stay unskilled.

Because human capital need long-term accumulation and is relatively stable in a short time (Sunde, 2005), firms can get some fixed information about current skill of each type of workers. We started from the firms in period t.

As mentioned above, the firm cannot get full information of each worker, the only information that the firm can get easily is the average performance in each category. So, some skilled workers will not be trusted, and some unskilled workers will be trusted. In the paper, the real problem is that there is a mismatch between tasks and workers, i.e. there are some old workers who are skilled will not get the important tasks they want, and some important tasks are allocated to unskilled workers. The mismatches can be shown in figure 2 .

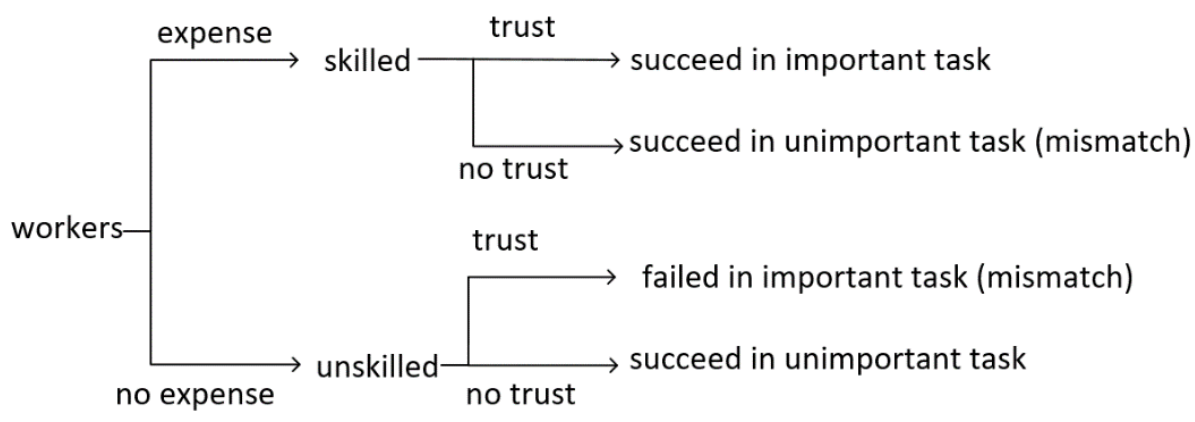

Figure 2. The mismatches in the labor force

Source: Drawn by the author

The firms are agents who pursuit of maximum profit. There are two kinds of tasks with different degrees of difficulties and importance. The degree of difficulties and importance are positively related. The firm decide if an important task should be allocated to a certain worker. If a worker is allocated an important task, he will get the high wage, no matter the task is done well or not. In the paper, I normalized that high wage to 1 . The worker can do well in unimportant tasks no matter he is skilled or not and he will only be paid the basic wage. Because the model focuses on the changes I 
normalize the basic wage to 0 .

If the important and difficult task is well done, the firm will get a total income larger than the high wage, that is $(1+\pi) . \pi$ here refers to the mark-up ratio of the firm. Because the high wage is normalized to $1, \pi$ is also the net profit of the firm after the high wage is paid. However, if the old worker fails to fulfill the important task, the firm will get nothing, but the firm still must keep the engagement and pay the old worker high wage 1.

What's more, the firm will suffer a loss due to the negative externalities of the failure. And the magnitude of the negative externalities will be multiplied by the degree of trust which this worker group get. Here the degree of trust is the probability a worker is allocated important skilled. Here we use $p_{t}$ to denote that probability and degree of trust, and $l p_{t}$ to denote the extra loss besides paying wages for failed tasks. So, the total loss should be $\left(-1-l p_{t}\right)$.

Why the loss is positively related to the degree of trust need to be explained further here. In evolutionary game theory and experimental economics, the notion of trust is much simpler: it is an expectation about another's behavior. And trust plays an important role in fostering cooperation (Acedo and Gomila, 2013). The higher possibility a certain kind of workers is allocated important task, namely the more trust the firm place on that kind of workers, will raise other workers' expectation about that kind of workers. Then more workers will be willing to cooperate with them and they are more likely to be placed at crucial position, like head derivatives trader, and there will be a closer relationship between their work and others', if they fail, the loss will be heavier (Greener, 2006). Although there will also be a positive externality if the old worker has done his important task well, the positive one is often relatively small, especially comparing to the negative one if he fails. So, the positive externality is not included in the discussion.

Human capital can't be accumulated in a short time, so we assume in period t, the group of workers have an average skill $\bar{s}_{t}$. And $\bar{s}_{t}$ will not change in period t.

Firms can get average skill of certain workers' group through methods such as sample surveys, which do not involve expensive costs. Because we have $\bar{s}_{t}$ fixed, so the firms can get that as certain information. The firm can maximize its profit function by choosing whether to allocate important tasks to each individual or not.

In our paper, all workers are divided into 6 groups: students in rich family, students in poor family, young worker with important task, young worker with unimportant task, old worker with important task, old worker with unimportant task. The students and their old worker parents are simultaneous. We will discuss the transactions among those groups more specifically in section 4. Readers who are confused about the division can refer to Figure 9 first. For simplicity, in the following discussion, we collectively call those workers and students as 'worker'.

As there are many workers in each group, and all the workers in one group are regarded as the same by the firm according to our assumption. The firm need to decide a proportion of workers to be allocated important tasks in each type. That proportion is the same to the probabilities that one worker is trusted with important task, that is $p_{t}$.

The firm's expected net profit is given by:

$$
\begin{aligned}
\mathrm{E}(\text { profit })_{t} & =p_{t}\left\{\pi \bar{s}_{t}+\left(-1-l p_{t}\right)\left(1-\bar{s}_{t}\right)\right\}+\left(1-p_{t}\right) * 0 \\
& =p_{t} \pi \bar{s}_{t}-p_{t}\left(1-\bar{s}_{t}\right)-p_{t}{ }^{2} l\left(1-\bar{s}_{t}\right)
\end{aligned}
$$

$p_{t}$ here means the proportion of workers that are trusted with important tasks. If we deem the total number of that kind of workers (such as old poor workers) as $1, p_{t}$ here is also the total amount of workers being trusted in that group (such as old poor workers). $\pi$ is the profit the firm can get if the important task is successful. $\bar{s}_{t}$ is the average skill level of workers in that group, and it is also the possibility of success. $\left(-1-l p_{t}\right)$ is the total loss of the firm, including paying wages for nothing and extra loss due to failure in cooperation. $\left(1-\bar{s}_{t}\right)$ is the possibility of failure. $\left(1-p_{t}\right)$ means the proportion of workers that are not trusted with important tasks. According to our assumption, firms will get no profit and no loss from unimportant tasks.

We use $p_{t}{ }^{*}$ to denote the ideal proportion of old workers to be allocated important tasks, for this proportion can generate the maximum expected profit.

$$
\frac{\partial \mathrm{E}(\text { profit })_{t}}{\partial p_{t}}=\pi \bar{s}_{t}-\left(1-\bar{s}_{t}\right)-2 p_{t} l\left(1-\bar{s}_{t}\right)
$$

If $\pi * \bar{s}_{t}-\left(1-\bar{s}_{t}\right) \leq 0$, that is $0 \leq \bar{s}_{t} \leq \frac{1}{1+\pi} \quad, \frac{\partial E(\text { profit })_{t}}{\partial p_{t}}<0$ when $p_{t} \in(0,1)$. So, $p_{t}^{*}=0$.

If $\pi * \bar{s}_{t}-\left(1-\bar{s}_{t}\right)>0$, we need further discussion:

$$
0<\frac{1}{1+\pi}<\frac{2 l+1}{(\pi+1+2 l)} \leq \bar{s}_{t} \leq 1,(\pi+1+2 \mathrm{l}) \bar{s}_{t} \geq 2 l+1,
$$




$$
\pi * \bar{s}_{t}-\left(1-\bar{s}_{t}\right) \geq 2 l\left(1-\bar{s}_{t}\right)>2 l\left(1-\bar{s}_{t}\right) p_{t}, \frac{\partial \mathrm{E}(\text { profit }) t}{\partial p_{t}}>0 \text { when } p_{t} \in(0,1) . \text { So, } p_{t}^{*}=1
$$

$$
0<\frac{1}{1+\pi} \leq \bar{s}_{t}<\frac{2 l+1}{(\pi+1+2 \mathrm{l})} \leq 1, \text { because } \frac{\partial^{2} \mathrm{E}(\text { profit })}{\partial\left(p_{t}^{2}\right)}=-2 l\left(1-\bar{s}_{t}\right)<0, \text { the firm will choose } p_{t}^{*} \text { to make }
$$

$$
\begin{aligned}
\frac{\partial \mathrm{E}(\text { profit }) t}{\partial p_{t}}=\pi \bar{s}_{t}-\left(1-\bar{s}_{t}\right)-2 l p_{t}\left(1-\bar{s}_{t}\right) & =0 \\
p_{t}{ }^{*} & =\frac{\pi}{2 l\left(1-\bar{s}_{t}\right)}-\frac{\pi+1}{2 l}
\end{aligned}
$$

In a word,

$$
p_{t}{ }^{*}=f\left(\bar{s}_{t}\right)=\left\{\begin{array}{cc}
0, & 0 \leq \bar{s}_{t}<\frac{1}{1+\pi} \\
\frac{\pi}{2 l\left(1-\bar{s}_{t}\right)}-\frac{\pi+1}{2 l}, & \frac{1}{1+\pi} \leq \bar{s}_{t}<\frac{2 l+1}{\pi+2 l+1} \\
1, & \frac{2 l+1}{\pi+2 l+1} \leq \bar{s}_{t} \leq 1
\end{array}\right.
$$

When $\frac{1}{1+\pi} \leq \bar{s}_{t}<\frac{2 l+1}{\pi+2 l+1}, f^{\prime}\left(\bar{s}_{t}\right)=\frac{\pi}{2 l} * \frac{1}{\left(1-\bar{s}_{t}\right)^{2}}>0, f^{\prime \prime}\left(\bar{s}_{t}\right)=\frac{\pi}{2 l} * \frac{2}{\left(1-\bar{s}_{t}\right)^{3}}>0$.

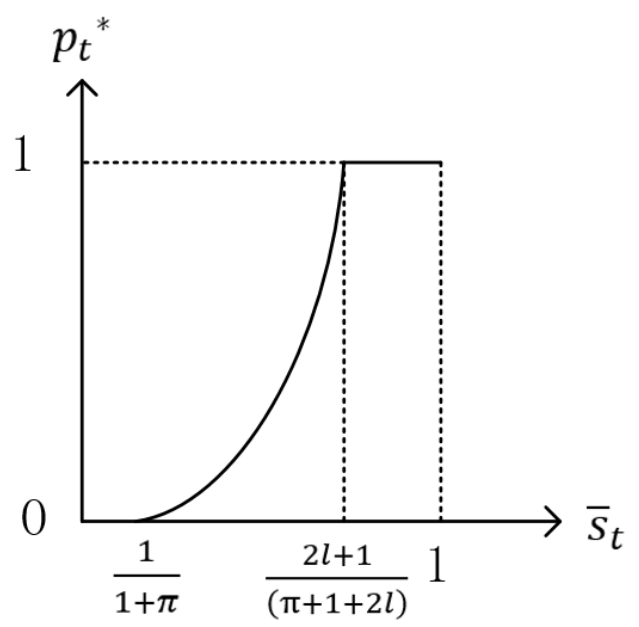

Figure 3. The illustration of function $p_{t}{ }^{*}=f\left(\bar{s}_{t}\right)$

Source: Drawn by the author

I assume workers are rational agents and a worker has a trade-off between becoming a skilled worker or not. However, that decision must be made in the previous period because human capital needs time to develop. As there are so many workers in the labor market, every single workers' decision will not change any property of that market. And every worker doesn't know other workers' decision when he makes his own.

Human capital can be regarded as commodity with extremely long production cycle. So, the Cobweb Theorem can be applied here. Cobweb Theorem explains why prices might be subject to periodic fluctuations in certain types of markets. It describes cyclical supply and demand in a market where the amount produced must be chosen before prices are observed. Producers' expectations about prices are assumed to be based on observations of previous prices (Ezekiel, 1938).

Here to apply the Cobweb model we must do some extension. In our model, the wage for important tasks is fixed and there is only the probability of being trusted with important tasks. This probability can be deemed as average price for labor. The higher the probability is, the more workers are willing to make some expense to become skilled.

The firm will probably discriminate the old worker by not giving him important tasks but that is not sure. There are many personal and opportunistic factors that may affect the outcome. For example, if the old worker has some good friends in the firm's managers, the firm will be clearer about his abilities and attitudes and perhaps will make a choice more based on his real abilities. There are so many such factors and they are random in the future period t. So, I should treat them as random disturbances. But the portion of workers who are allocated important task $p_{t-1}{ }^{*}$ is decided. 
The information is asymmetry: The old worker can get nearly full information about the attitude of the firm before he decides what to do. He will know the exact probability of being discriminated in period t-1. However, he will not know whether he will be discriminated or not in period $t$ for sure because all he gets is just an outdated probability. A firm can get information about the general skill level of workers, but getting each individual's exact skill level is too costly.

In the beginning of a period, the worker must decide whether to be skilled or not. If he has made his choice, he cannot change it. This assumption is based on the following fact: the time and money they spend on training are sunk costs. If a worker first decides to be skilled then he regrets for his choice, he will find it is too late. The expense to be skilled is denoted by e.

The workers with important tasks are usually respected by the society, that make them value their reputation. If a worker fails an important task, especially when his incompetence becomes a common knowledge, he will feel humiliation. Hierarchy of needs theory suggests that esteem needs is an important need for human being (Maslow, 1943). Although this phenomenon is hardly discussed in economic studies, it may even cause serious psychology problems (Brodsky, 1971). Because Confucianism stresses the traditional boundaries of ethical responsibility (Park and Chesla, 2007), the humiliation effect due to incompetence in the East Asian countries will heavier. The humiliation coefficient $\lambda$ reflects the negative utility if he fails. $\lambda$ is highly related to the culture, and in the same culture, all old workers have similar humiliation factor, while it differs greatly in different cultures.

Because workers pay the expense in the previous period, we need to introduce the discount factor $\beta \in[0,1]$ to transfer that expense into current period value. Higher $\beta$ means more patience. The discount factor here reflects individuals' patience, which also has a close relation with culture. For example, Confucianism stresses moderation and patience (Chen and Chung, 1994), namely, pays more attention to the future. That means a bigger $\beta$.

We assume a skilled worker, if doesn't get important tasks in a period, will not get any advantage in becoming skilled in the next period. That assumption will make the utility in each period independent to each other.

So, the decision of the old worker in period $\mathrm{t}-1$ is made through three steps.

a. He will get the probability of being given important task $p_{t-1}{ }^{*}$ and not being given important task $\left(1-p_{t-1}{ }^{*}\right)$.

b. He will calculate the expected utility when he becomes skilled $V_{t}^{s}$ and when he becomes unskilled $V_{t}^{u}$. $\beta$ here is the discount factor, reflecting patience of that worker, $0<\beta<1$, implying the same amount of expense in period $t-$ 1 worth more than in period $t$.

$$
V_{t}^{s}=p_{t-1}^{*}-\frac{e}{\beta}
$$

$p_{t-1}{ }^{*}$ here is the expected return from high wages for being trusted with important tasks. $e$ is the expense to be skilled. Because it need to be paid one period before, so it should be discounted to current value.

$$
V_{t}^{u}=p_{t-1}^{*}(1-\lambda)
$$

$p_{t-1}{ }^{*}$ here is also the expected return from high wages for being trusted with important tasks. 1 is the wage he gets from the firm. But as he failed the task, so it is shameful to receive that kind of money. $\lambda$ here reflects how proud he is. If he is care about his fame, that failure will make him feel bad. So, a proportion of positive utility brought by the high wage will be cancled by this bad feeling.

c. He will compare the expected utility of being skilled and not being skilled, and then he makes the choice.

$$
V_{t}^{s}-V_{t}^{u}=\lambda p_{t-1}^{*}-\frac{e}{\beta}
$$

The workers must spend different expenses to be skilled. We assume these expenses satisfies uniform distribution between 0 and $2 \bar{e}$. The probability density function is given by:

$$
g(e)=\frac{1}{2 \bar{e}}, e \in[0,2 \bar{e}]
$$

If

$$
\lambda p_{t-1}^{*}-\frac{e}{\beta}=0
$$

He will be indifferent. The indifferent old worker has the critical expense:

$$
e^{*}=\lambda \beta p_{t-1}^{*}
$$

If $\mathrm{e}<e^{*}$, he will choose to be skilled. 
If e $>e^{*}$, he will choose to be unskilled.

Then the average skill, namely, current proportion of skilled workers is:

$$
\bar{s}_{t}=\int_{0}^{\lambda \beta p_{t}{ }^{*}} \frac{1}{2 \bar{e}} d e=\left\{\begin{array}{c}
\frac{\lambda \beta p_{t-1}{ }^{*}}{2 \bar{e}}, 0 \leq p_{t-1}{ }^{*} \leq \frac{2 \bar{e}}{\lambda \beta} \\
1, p_{t-1}{ }^{*}>\frac{2 \bar{e}}{\lambda \beta}
\end{array}\right.
$$

$\mathrm{f}(\mathrm{e})$

0

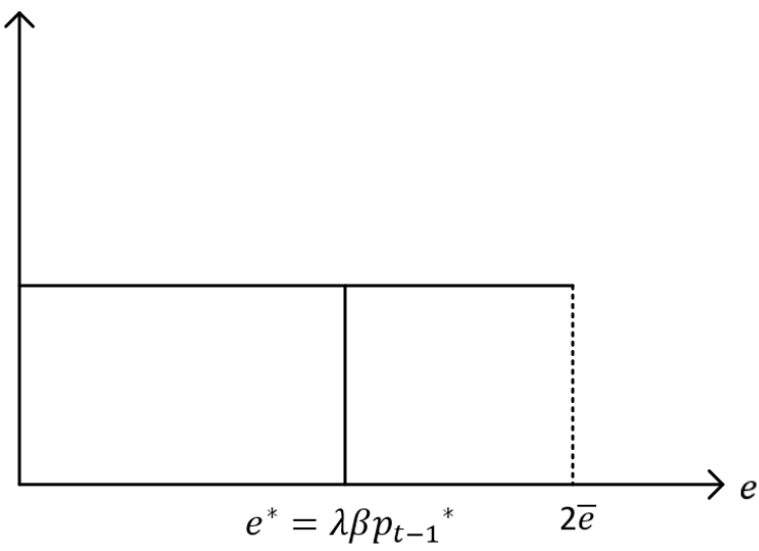

Figure 4. The choice of workers

Source: Drawn by the author

\section{Equilibrium Analysis}

Definition 1. An equilibrium is a sequence $\left\{s_{t}\right\}_{t=1}^{\infty}$ with the initial condition $s_{t} \in[0,1]$ and time invariant average expense to be skilled $\bar{e}>0$ that:

a. The proportion of old workers allocated important task is given by Eq. (4).

b. The current proportion of skilled workers is given by Eq. (11).

Assumption $1 \lambda \in\left(0, \frac{1}{\beta}\right)$

Here, we assume $\lambda \in\left(0, \frac{1}{\beta}\right)$ to exclude the extremely high self-esteem or no self-esteem.

If $\lambda \beta p_{t-1}{ }^{*}>2 \bar{e}$, that means all workers will choose to be skilled. Because $0 \leq p_{t-1}{ }^{*} \leq 1$, to make that possible, at least we need $\lambda \beta>2 \bar{e}$. This means either the worker has relatively high self-esteem that he can't afford to be humiliated, or the average expense to be skilled is relatively low, or he has more patience.

If we substitute $p_{t-1}{ }^{*}$ in (11) with (4). Under the condition $\lambda \beta>2 \bar{e}$ we get

$$
\begin{aligned}
& \bar{s}_{t}=\left\{\begin{array}{cc}
0, & 0 \leq \bar{s}_{t-1}<\frac{1}{1+\pi} \\
\frac{\lambda \beta}{2 \bar{e}} *\left[\frac{\pi}{2 l\left(1-\bar{s}_{t-1}\right)}-\frac{\pi+1}{2 l}\right], & \frac{1}{1+\pi} \leq \bar{s}_{t-1}<\Omega \\
1, & \Omega \leq \bar{s}_{t-1} \leq 1
\end{array}\right. \\
& \Omega=1-\frac{\pi}{2 l\left[\frac{2 \bar{e}}{\lambda \beta}+\frac{\pi+1}{l}\right]}
\end{aligned}
$$

Lemma 1. When $\frac{\lambda \beta}{2 \bar{e}}>1$, there are two stable steady-state equilibria:

$$
\begin{aligned}
& \bar{s}_{t}=0, p_{t}{ }^{*}=0 ; \\
& \bar{s}_{t}=1, p_{t}{ }^{*}=1
\end{aligned}
$$

And an unstable stable steady-state equilibrium: 


$$
\begin{gathered}
\bar{s}_{t}=\Phi, p_{t}{ }^{*}=\frac{\pi}{2 l(1-\Phi)}-\frac{\pi+1}{2 l} \\
\Phi=\frac{4 \bar{e} l-\pi \lambda \beta-\lambda \beta+\sqrt{(4 \bar{e} l-\pi \lambda \beta-\lambda \beta)^{2}+16 \bar{e} l \beta \lambda^{2}}}{8 \bar{e} l}
\end{gathered}
$$

Proof 1. From E.q.(12), E.q.(4) and the figure 5, it is easy to find the two stable steady-state equilibria.

when $\frac{1}{1+\pi} \leq \bar{s}_{t-1}<\Omega, \quad \bar{s}_{t}=\frac{\lambda \beta}{2 \bar{e}} *\left[\frac{\pi}{2 l\left(1-\bar{s}_{t}\right)}-\frac{\pi+1}{2 l}\right]$, we get

$\bar{s}_{t}=\frac{4 \bar{e} l-\pi \lambda \beta-\lambda \beta+\sqrt{(4 \bar{e} l-\pi \lambda \beta-\lambda \beta)^{2}+16 \bar{e} l \beta \lambda^{2}}}{8 \bar{e} l}=\Phi>0 . \quad p_{t}^{*}=\frac{\pi}{2 l(1-\Phi)}-\frac{\pi+1}{2 l}$

The smaller $\bar{s}_{t}$ is dropped because it is negative.

And we can find that steady state is unstable from the figure.

Q.E.D.

Figure 5 illustrates the graph. The area marked by (L1) in Figure 8 indicates the set of parameters $\left(\lambda, \frac{2 \bar{e}}{\beta}\right)$ satisfying the condition above.

If we substitute $p_{t}{ }^{*}$ in (11) with (4). Under the condition $\lambda \leq \frac{2 \bar{e}}{\beta}$ we get

$$
\bar{s}_{t}=\left\{\begin{array}{cc}
0, & 0 \leq \bar{s}_{t-1}<\frac{1}{1+\pi} \\
\frac{\lambda \beta}{2 \bar{e}} *\left[\frac{\pi}{2 l\left(1-\bar{s}_{t-1}\right)}-\frac{\pi+1}{2 l}\right], & \frac{1}{1+\pi} \leq \bar{s}_{t-1}<\frac{2 l+1}{\pi+2 l+1} \\
\frac{\lambda \beta}{2 \bar{e}}, & \frac{2 l+1}{\pi+2 l+1} \leq \bar{s}_{t-1} \leq 1
\end{array}\right.
$$

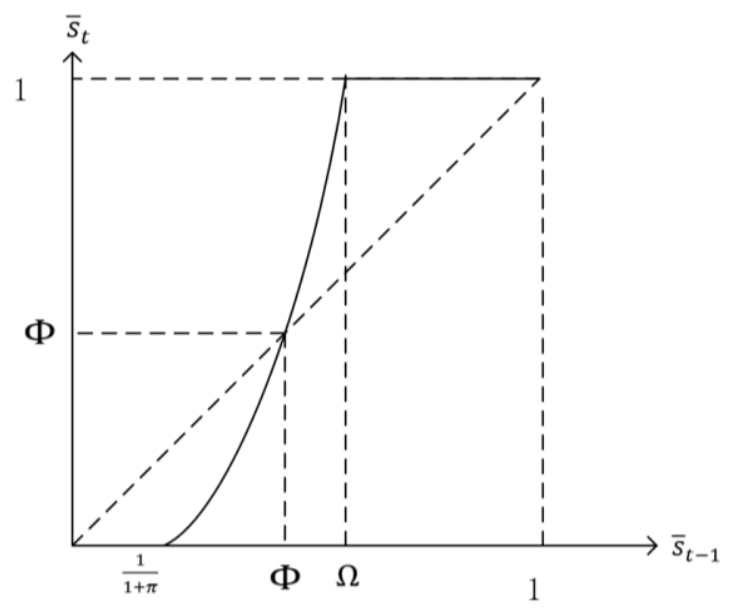

Figure 5. Dynamic motion of the proportion of skilled old worker when $\frac{\beta \lambda}{2 \bar{e}}>1$

Source: Drawn by the author

There are two scenarios which are illustrated in Figure 6 and Figure 7.

Lemma 2. When $0<\frac{\lambda \beta}{2 \bar{e}} \leq \frac{2 l+1}{\pi+2 l+1}$, there is only one stable equilibrium: $\bar{s}_{t}=0, p_{t}{ }^{*}=0$.

Proof 2. From E.q.(13), E.q.(4) and Figure 6, it is easy to find there is only one stable equilibrium:

$$
\bar{s}_{t}=0, p_{t}^{*}=0 \text {. }
$$

Q.E.D. 


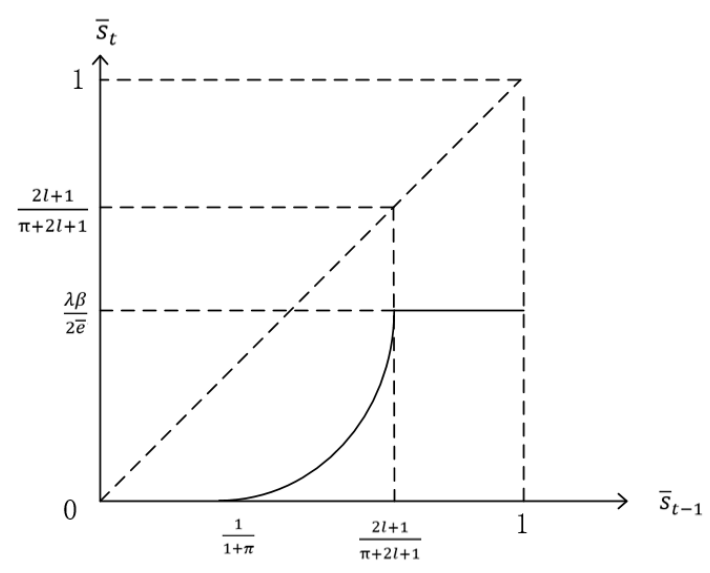

Figure 6. Dynamic motion of the proportion of skilled old worker when $0<\frac{\lambda \beta}{2 \bar{e}} \leq \frac{2 l+1}{\pi+2 l+1}$

Source: Drawn by the author

Lemma 3. When $\frac{2 l+1}{\pi+2 l+1}<\frac{\lambda \beta}{2 \bar{e}} \leq 1$, there are two stable equilibria:

$$
\begin{gathered}
\bar{s}_{t}=0, p_{t}^{*}=0 ; \\
\bar{s}_{t}=\frac{\lambda \beta}{2 \bar{e}}, p_{t}^{*}=1
\end{gathered}
$$

And an unstable equilibrium:

$$
\bar{s}_{t}=\Phi=\frac{4 \bar{e} l-\pi \lambda \beta-\lambda \beta+\sqrt{(4 \bar{e} l-\pi \lambda \beta-\lambda \beta)^{2}+16 \bar{e} l \beta \lambda^{2}}}{8 \bar{e} l}>0, p_{t}^{*}=\frac{\pi}{2 l(1-\Phi)}-\frac{\pi+1}{2 l}
$$

Proof 3. From E.q.(13), E.q.(4) and Figure 7, it is easy to find there are two stable equilibria:

$$
\begin{gathered}
\bar{s}_{t}=0, p_{t}{ }^{*}=0 ; \\
\bar{s}_{t}=\frac{\lambda \beta}{2 \bar{e}}, p_{t}{ }^{*}=1 \\
\text { When } \frac{1}{1+\pi} \leq \bar{s}_{t-1}<\frac{2 l+1}{\pi+2 l+1}, \bar{s}_{t}=\frac{\lambda \beta}{2 \bar{e}} *\left[\frac{\pi}{2 l\left(1-\bar{s}_{t}\right)}-\frac{\pi+1}{2 l}\right], \text { we get } \\
\bar{s}_{t}=\frac{4 \bar{e} l-\pi \lambda \beta-\lambda \beta+\sqrt{(4 \bar{e} l-\pi \lambda \beta-\lambda \beta)^{2}+16 \bar{e} l \beta \lambda^{2}}}{8 \bar{e} l}=\Phi>0, p_{t}{ }^{*}=\frac{\pi}{2 l(1-\Phi)}-\frac{\pi+1}{2 l}
\end{gathered}
$$

The smaller $\bar{s}_{t}$ is dropped because it is negative.

And we can find that steady state is unstable from Figure 7.

Q.E.D.

Figure 7 illustrates the graph of the above scenario. The area marked by (L2) in Figure 8 indicates the set of parameters $\left(\lambda, \frac{2 \bar{e}}{\beta}\right)$ satisfying the condition in Lemma 3. The area marked by (L3) in Figure 8 indicates the set of parameters $(\lambda$, $\frac{2 \bar{e}}{\beta}$ ) satisfying the condition in Lemma 2 .

From the analysis above, we can get the conclusion that there always exists a poverty trap which will make the proportion of skilled workers become 0 . In the situation of Lemma 1 and Lemma 3, a transitory change of $\bar{s}_{t}$ can overcome that poverty trap; in the situation of Lemma 2 , the poverty trap can't be overcome by transitory change of $\bar{s}_{t}$. 


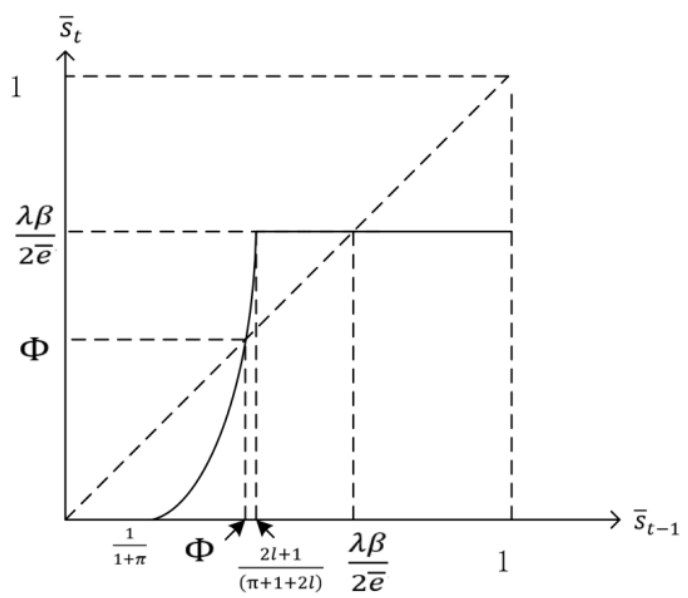

Figure 7. Dynamic motion of the proportion of skilled old worker when $\frac{2 l+1}{\pi+2 l+1}<\frac{\lambda \beta}{2 \bar{e}} \leq 1$

Source: Drawn by the author

Assumption 2. The government will try to draw the economy out of the poverty trap. If any other stable equilibrium is possible, the government will help workers escape the poverty trap through transitory policies.

For example, the government can compensate the expense to be skilled by providing public training program or giving them subsidies for being skilled. In extreme situations, the compensation can be higher than the expense. But continuous compensation costs too much that it is difficult for the national finance to support it.

The steady state equilibria here can reflect the tendency, but the positions in the equilibria are perfect states where the economy can hardly reach in real world. There are two reasons. First, the formation of human capital is a long process which usually falls behind the market. Second, any shock such as policy change and technology progress can easily change the coefficients in our model.

We can illustrate the equation $\lambda=\frac{2 \bar{e}}{\beta}$ and $\lambda=\frac{2 \bar{e}}{\beta} * \frac{2 l+1}{\pi+1+2 l}=\frac{2 \bar{e}}{\beta} * \frac{1+2 l}{\pi+1+2 l}$ in the following figure to make the analysis easier. We can see $\lambda=\frac{2 \bar{e}}{\beta}$ and $\lambda=\frac{2 \bar{e}}{\beta} * \frac{1+2 l}{\pi+1+2 l}$ divide the whole area $\left(\lambda \in\left(0, \frac{1}{\beta}\right), \frac{2 \bar{e}}{\beta} \in(0,+\infty)\right)$ into three parts with respective stable state equilibria:

$(\mathrm{L} 1): \bar{e} \in\left(0, \frac{\lambda \beta}{2}\right)$, all skilled, all trusted.

(L2): $\bar{e} \in\left(\frac{\lambda \beta}{2}, \frac{\lambda \beta}{2} * \frac{\pi+1+2 l}{2 l+1}\right)$, part skilled, all trusted.

(L3): $\bar{e} \in\left(\frac{\lambda \beta}{2} * \frac{\pi+1+2 l}{2 l+1},+\infty\right)$, all unskilled, all not trusted.

For any given $\frac{2 \bar{e}}{\beta}$ below $\frac{\pi}{1+2 l}+1$, higher $\lambda$ is related to higher probability of all skilled equilibrium (L1) or partial skilled equilibrium (L2) and lower probability of all unskilled equilibrium (L3). In (L2), this means higher proportion of skilled old workers: $\bar{s}_{t}=\frac{\lambda \beta}{2 \bar{e}}$.

This means in a society with high self-esteem culture will enjoy a higher proportion of skilled workers and trust workers more. In Confucian culture, 'face' can be defined as an individual's contingent self-esteem (Ng, 2001). An individual will feel 'lose face' when his failure was exposed in public (Hwang, 2006). This will contribute to a high proportion of skilled workers and better wages for them in some east Asian countries, like Japan.

Other things being equal, a bigger discount factor $\beta$ means a lower $\frac{2 \bar{e}}{\beta}$, so it is also related to higher probability of all 
skilled equilibrium (L1) or partial skilled equilibrium (L2) and lower probability of all unskilled equilibrium (L3). In (L2), this also means higher proportion of skilled old workers: $\bar{s}_{t}=\frac{\lambda \beta}{2 \bar{e}}$. Confucianism stresses moderation and patience (Chen and Chung, 1994), namely, pays more attention to the future. That means a bigger $\beta$ in Confucian countries. This will also contribute to a high proportion of skilled workers and better wages for them in those countries.

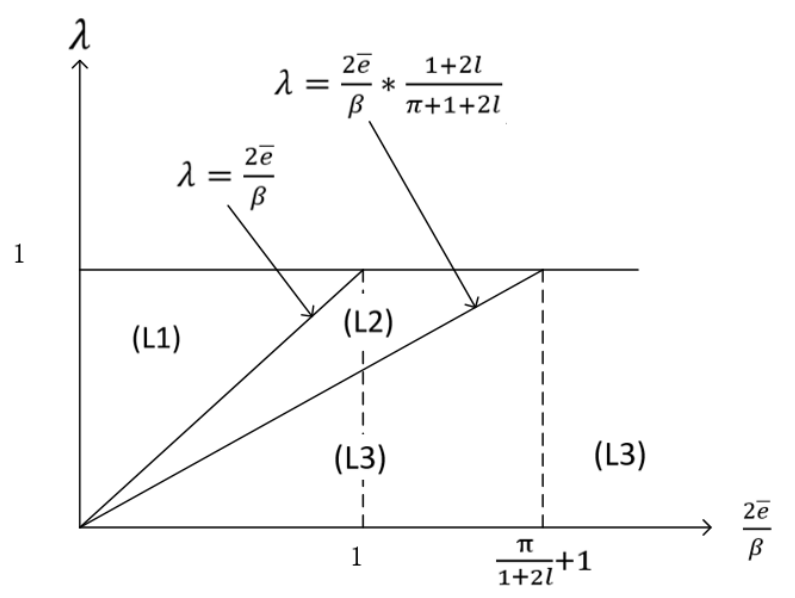

Figure 8. Existences and properties of steady-state equilibria

Source: Drawn by the author

\section{General Analysis}

To take full advantage of our model, we will use our model to do some general analysis and try to find the transactions among different types of workers: rich students, poor students, rich young workers, poor young workers, rich old workers and poor old workers.

At first, we need to clarify how many periods in a worker's whole life need to be considered. In this paper, we have three periods: student period, young worker period and old worker period. The years before schooling and the years after retirement are not included in our discussion.

The 'rich' and 'poor' mentioned above refers to workers status during a certain period, not at the beginning of a certain period. And they are the results of being trusted or not in the beginning of a period. To avoid confusion, we must make it clear that rich young workers mentioned above refers to the individuals, no matter rich students or poor students, that are trusted with important tasks in the beginning of his young career and remain rich until the end of their young career. And rich old workers mentioned above refers to the individuals that are trusted with important tasks in the beginning of his old career and remain rich until the end of their old career, and their children are rich students. Vice versa.

In the student period, a student will make his choice on education and training based on his expectation of next period (Sunde, 2005), which correspondent to his young worker period. In the young worker period, a young worker will make his choice on education and training based on his expectation of next period, which correspondent to his old worker period.

We assume each parent has a child in average. As we here discuss situations in developed countries where late marriage and late motherhood are widespread in recent years (Retherford et al., 2001; Jones, 2007, 2012), we assume a parent's old worker period is correspondent to his child's student period. That means a rich old worker is correspondent to a rich student and a poor old worker is correspondent to a poor student.

Some researchers have found rich-born individuals have an advantage over poor-born individuals in being educated or trained (Becker and Tomes, 1979; Solon, 2004; Blumkin and Sadka, 2005; Hassler et al., 2007a). For example, rich agents may have a home environment more suitable for education, along with better knowledge of things naturally provided to their children. In other words, comparing to the rich, poor individuals have disadvantages in those aspects. Even a very high ability poor individual will be affected because he can do better if he is rich. In our paper, we use a multiplier $\varphi^{p}>1$ to measure that disadvantage. If a rich individual need to spend e to become skilled, every poor individual with the same intelligence and age will have to spend $\varphi^{p} * \mathrm{e}$.

Similarly, Similarly, due to deteriorative health, individuals have an disadvantage in maintaining skill when they turn old (McArdle et al., 2002). We use $\varphi^{o}>1$ to measure that disadvantage to maintain skill due to old age. If a student need to spend e in his student period to become a skilled young worker, then a young worker with the same intelligence 
and wealth status will have to spend $\varphi^{p} * \mathrm{e}$ in his young worker period to become a skilled old worker.

First, we set the rich students' average expense to be skilled young workers as standard: $\bar{e}^{r y}=\bar{e}_{0}$.

Then we use $\varphi^{p}>1$ to measure the disadvantage due to poverty and $\varphi^{o}>1$ to measure the disadvantage due to aging. Both will make the average expense increase.

Then the poor students' average expense to be skilled young workers: $\bar{e}^{p y}=\varphi^{p} \bar{e}_{0}$.

The rich young workers' average expense to be skilled old workers: $\bar{e}^{r o}=\varphi^{o} \bar{e}_{0}$.

The poor young workers' average expense to be skilled old workers: $\bar{e}^{p o}=\varphi^{o} \varphi^{p} \bar{e}_{0}$.

In this paper, we discuss the discriminations of the old and the poor. So, we assume when rich students step into workplace, they are all well prepared for important tasks, so $\bar{e}_{0} \in\left(0, \frac{\lambda \beta}{2}\right)$. He will not suff er discrimination. So, the transaction from rich students to rich young workers is $100 \%$. We assume other groups are not all prepared for important tasks. On the other hand, when poor young workers grow old, the discriminations will deteriorate greatly. We assume $\varphi^{o} \varphi^{p} \bar{e}_{0} \in\left(\frac{\lambda \beta}{2} * \frac{\pi+1+2 l}{2 l+1},+\infty\right)$, That means without government help, the average skill of old poor will deteriorate to a degree that none of them will be trusted with important tasks. Just as shown in the Figure 9, Figure 10, Figure 11 and Figure 12, there are four possible scenarios. At first, we will discuss two special situations and make some plausible explanation and then we will discuss two more general situations and find the reason of low intergenerational mobility (class solidification) based on those discussions. The transaction will be shown by the arrows. The dashed line arrows represent those transaction will be harder and harder as each type move towards stable steady-state equilibrium.

First, when

$$
\begin{aligned}
\bar{e}^{r y} & =\bar{e}_{0} \in\left(0, \frac{\lambda \beta}{2}\right), \bar{e}^{p y}=\varphi^{p} \bar{e}_{0} \in\left(\frac{\lambda \beta}{2} * \frac{\pi+1+2 l}{2 l+1},+\infty\right), \\
\bar{e}^{r o} & =\varphi^{o} \bar{e}_{0} \in\left(\frac{\lambda \beta}{2} * \frac{\pi+1+2 l}{2 l+1},+\infty\right), \bar{e}^{p o}=\varphi^{o} \varphi^{p} \bar{e}_{0} \in\left(\frac{\lambda \beta}{2} * \frac{\pi+1+2 l}{2 l+1},+\infty\right) .
\end{aligned}
$$

The transactions are shown in Figure 9. We can see there is a poverty trap. The rich will be less and less while the poor will be more and more. That situation is extremely rare when we consider the whole economy, especially in current developed countries. However, there are some professions that may be dangerous and harmful to health, and those professions require the workers to be young, such as firemen and miners. So rich students know what they will face in the beginning so most of them will not choose to do those jobs at the first place.

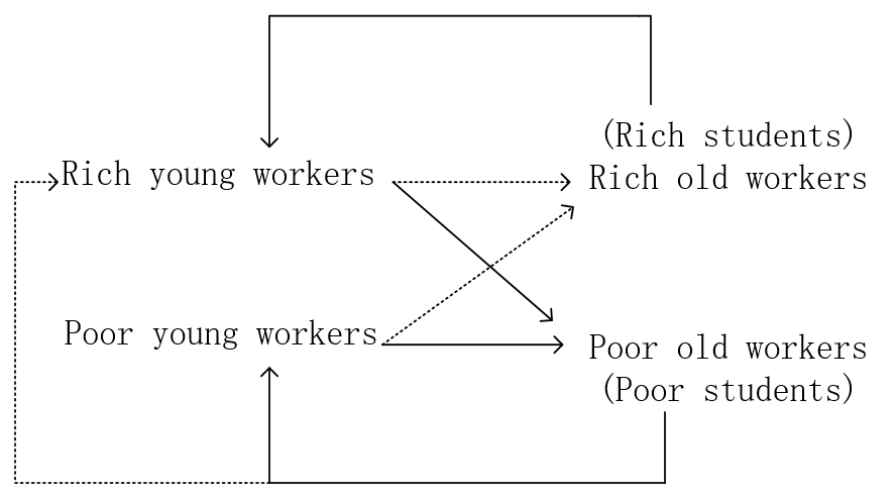

Figure 9. The transaction of different groups (poverty trap)

Source: Drawn by the author

Second, when

$$
\bar{e}^{r y}=\bar{e}_{0} \in\left(0, \frac{\lambda \beta}{2}\right), \bar{e}^{p y}=\varphi^{p} \bar{e}_{0} \in\left(\frac{\lambda \beta}{2}, \frac{\lambda \beta}{2} * \frac{\pi+1+2 l}{2 l+1}\right),
$$




$$
\bar{e}^{r o}=\varphi^{o} \bar{e}_{0} \in\left(\frac{\lambda \beta}{2} * \frac{\pi+1+2 l}{2 l+1},+\infty\right), \bar{e}^{p o}=\varphi^{o} \varphi^{p} \bar{e}_{0} \in\left(\frac{\lambda \beta}{2} * \frac{\pi+1+2 l}{2 l+1},+\infty\right) .
$$

The transactions are shown in Figure 10. We can see rich young workers and poor old workers tend to become the main part of workforce. That situation is also extremely rare when we consider the whole economy, especially in current developed countries. However, there are some professions that require the workers to be young, and those professions may accompany with high wages, but only for young workers. The represent of those professions are fashion models. All say that fashion modelling is a career for the young. Clever fashion models will plan their future well. Most of those workers will quit that profession before they turn old.

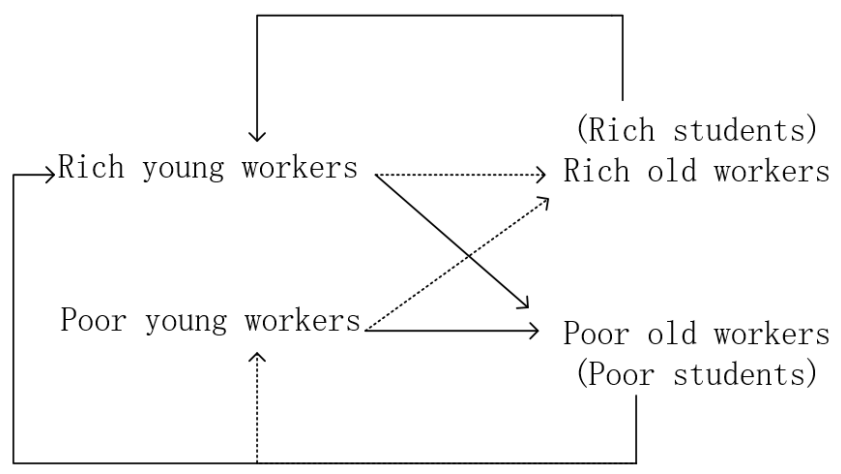

Figure 10. The transaction of different groups (career for the young)

Source: Drawn by the author

Third, when

$$
\begin{gathered}
\bar{e}^{r y}=\bar{e}_{0} \in\left(0, \frac{\lambda \beta}{2}\right), \bar{e}^{p y}=\varphi^{p} \bar{e}_{0} \in\left(\frac{\lambda \beta}{2} * \frac{\pi+1+2 l}{2 l+1},+\infty\right), \\
\bar{e}^{r o}=\varphi^{o} \bar{e}_{0} \in\left(\frac{\lambda \beta}{2}, \frac{\lambda \beta}{2} * \frac{\pi+1+2 l}{2 l+1}\right), \bar{e}^{p o}=\varphi^{o} \varphi^{p} \bar{e}_{0} \in\left(\frac{\lambda \beta}{2} * \frac{\pi+1+2 l}{2 l+1},+\infty\right) .
\end{gathered}
$$

The transactions are shown in Figure 11. This situation is common in many developed countries. We can find a class solidification: rich (poor) individuals tend to remain rich all their lives and have rich (poor) children and rich (poor) grandchildren. In some stable societies without turmoil, class solidification usually becomes more and more serious. For example, some researchers find America social mobility is declining (Cowen, 2017). In our model, that means in some stable societies, people can have stable expectations which will make each group move towards stable equilibrium more smoothly. One important reason is the increasing education expenses. If the inequality of education and training is big enough, the poor will suff er great disadvantage in education and training cost. And then the discriminations will be so heavy that the poor find it is hard to find a good job even in the start point of their career. As they turn old, things become worse. Poor individuals will have less opportunity and motivation to move upward (although for the technical progress their current living standard may be much better than the rich in the past). Even though poor students can receive better education in the help of student loan, they will find it is hard to cover their time and money cost by wages, especially when the hope of finding high wage jobs is dim.

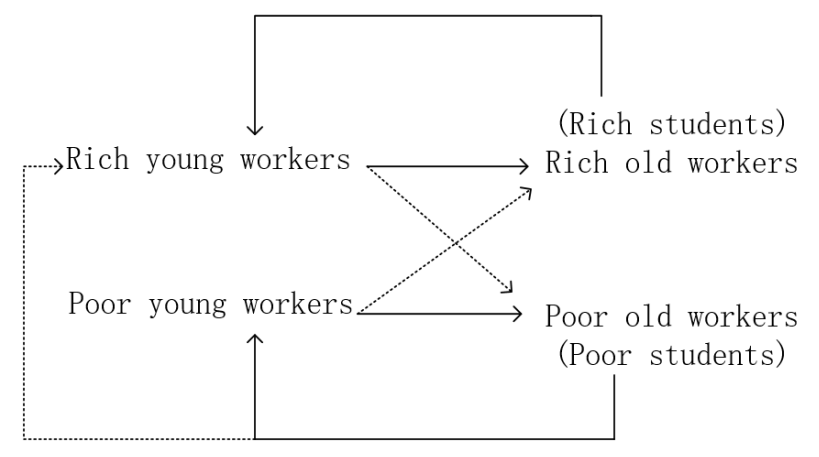

Figure 11. The transaction of different groups (class solidification)

Source: Drawn by the author 
Fourth, when

$$
\begin{gathered}
\bar{e}^{r y}=\bar{e}_{0} \in\left(0, \frac{\lambda \beta}{2}\right), \bar{e}^{p y}=\varphi^{p} \bar{e}_{0} \in\left(\frac{\lambda \beta}{2}, \frac{\lambda \beta}{2} * \frac{\pi+1+2 l}{2 l+1}\right), \\
\bar{e}^{r o}=\varphi^{o} \bar{e}_{0} \in\left(\frac{\lambda \beta}{2}, \frac{\lambda \beta}{2} * \frac{\pi+1+2 l}{2 l+1}\right), \bar{e}^{p o}=\varphi^{o} \varphi^{p} \bar{e}_{0} \in\left(\frac{\lambda \beta}{2} * \frac{\pi+1+2 l}{2 l+1},+\infty\right) .
\end{gathered}
$$

The transactions are shown in Figure 12. We can find poor students can become rich young workers if their disadvantages are small enough. That means in a society with relatively high quality and equal education resources, generation mobility will be higher and common prosperity is easier to achieve. That is more common in some Nordic European countries. In the development of Four Asian Tigers, an abundant public education and training resources also play a key role, especially Singapore. Confucianism put emphasis on education and regard educating people as a responsibility of countries and authorities (Marginson, 2011). That explains why east Asian countries usually enjoy a better public education and training system during their development. According to our analysis, this will lead to higher social mobility and prosperity.

Those four kinds of transactions usually exist in the same country and in the same time. The proportion of each transaction will determine the countries' general situation.



Figure 12. The transaction of different groups (common prosperity)

Source: Drawn by the author

\section{Concluding Remarks}

Now it is time to solve the four questions we raised in the introduction.

First, what is the economic reason that some individuals choose not to be skilled when they can afford the expense in the help of loans and it seems profitable? Because individuals are rational agents who try to maximize their expected utilities. Being skilled is not the same as being rich because he is not sure whether he can get high wages. And when the expense to be skilled is too high, even high wage will not cover the cost. So, remaining unskilled is a rational choice for some individuals. Sometimes, being skilled seems profitable but in fact is not profitable because he may not get the work he wants. In our model, being skilled will not increase the probability of finding a good job. Although this assumption is a little extreme, but mismatch in work place is a common thing, and a lot of people are not satisfied with their jobs. So being unskilled may be a better choice for some individuals.

Second, why individuals choose various kind of education and training with various prices? Because individuals' intelligence varies and nowadays education and training become more and more flexible. If they decided to become skilled, individuals who have full knowledge of themselves will choose the least amount of education or training expense that will make them skilled. That is an important reason why personalization in education and training becomes a new global trend.

Third, what determines the evolution of a certain type of workers' performance? And fourth, what determines whether the discrimination for a certain type of workers will deteriorate? It depends on the relative sizes of workers' average expense to be skilled, workers' self-esteem in that culture and the discount rate. Given other conditions remain unchanged, the average expense to be skilled plays a key role. The workers' performance and discrimination will interact with each other. If that type of workers have relatively low expense to be skilled, the discriminations towards them will tend to reduce and workers' performance will be better, otherwise the discrimination tend to increase, and workers' performance will be worse.

Fifth, what is the relationship between the Confucianism and East Asian economic miracle? There are three reasons. First, Confucianism usually accompany with high self-esteem, so the Gresham's Law, namely, bad money drives out good, will do less harm in Confucianism countries. Second, Confucianism emphasis patience and moderation, that will 
make the agents be more patient, which will the expense to be skilled less unpleasant to the current individuals. Third, Confucianism regard education as a responsibility, both for the individuals and authorities, that will lead to a better and more equal public education and training system, which is essential to the economic development in those countries.

Finally, why in most developed countries, after the second world war, intergenerational mobilities tend to reduce? When the social is stable, people can form more specific expectations of the future, so the process to steady state equilibrium will be smoother. If educational and training resource in that society is unfair enough to the poor, then the closer the work force is to the steady state equilibrium, the less upwards opportunities poor individuals will have.

There are at least two limitations of our current research.

First, for the simplicity of analysis and calculation, we assume all the workers' expenses to be skilled satisfies uniform distribution. A distribution with fewer extreme cases such as normal distribution is closer to the reality.

Second, in our analysis, a skilled worker is assumed to share the same opportunities with others in the same group. In the real world, skilled workers can generate some signals to the firm, which will bring him more opportunities.

Both limitations need further discussion in our future research.

\section{Acknowledgements}

The author would like to thank two anonymous referees for their valuable comments and suggestions.

\section{References}

Acedo, C., \& Gomila, A. (2013). Trust and cooperation: a new experimental approach. Annals of the New York Academy of Sciences, 1299(1), 77-83. https://doi.org/10.1111/nyas.12142

Akerlof, G. (1970).The market for "lemons": Quality uncertainty and the market mechanism. The quarterly journal of economics, 488-500. https://doi.org/10.2307/1879431

Antonenko, D., \& Flöel, A. (2014). Healthy aging by staying selectively connected: a mini-review. Gerontology, 60(1), 3-9. https://doi.org/10.1159/000354376

Arawatari, R., \& Ono, T. (2013). Inequality, mobility and redistributive politics. Journal of Economic Theory, 148(1), 353-375. https://doi.org/10.1016/j.jet.2012.12.010

Arawatari, R., \& Ono, T. (2015). A Political Economy Model of Earnings Mobility and Redistribution Policy. Journal of Public Economic Theory, 17(3), 346-382. https://doi.org/10.1111/jpet.12112

Arawatari, R., \& Ono, T. (2015). Inequality, mobility and redistributive taxation in a finance-constrained economy. Applied Economics and Finance, 2(4), 143-159. https://doi.org/10.11114/aef.v2i4.1174

Arawatari, R., \& Ono, T. A. (2009). Second chance at success: a political economy perspective. Journal of Economic Theory, 144(3), 1249-1277. https://doi.org/10.1016/j.jet.2008.11.002

Avery, C., \& Turner, S. (2012). Student loans: Do college students borrow too much or not enough? The Journal of Economic Perspectives, 26(1), 165-192. https://doi.org/10.1257/jep.26.1.165

Bastos, J. L., Barros, A, D., Celeste, R. K., Paradies, Y., \& Faerstein, E. (2014). Age, class and race discrimination: their interactions and associations with mental health among Brazilian university students. Cadernos de saude publica, 30(1), 175-186. https://doi.org/10.1590/0102-311X00163812

Becker, G. S., \& Tomes, N. (1979). An equilibrium theory of the distribution of income and intergenerational mobility. Journal of political Economy, 87(6), 1153-1189. https://doi.org/10.1086/260831

Blumkin, T., \& Sadka, E. (2005). Income taxation and wage policy: an application to minimum wage. International Tax and Public Finance, 12(6), 713-722. https://doi.org/10.1007/s10797-005-0412-0

Brodsky, C, M. (1971). Social psychiatric consequences of job incompetence. Comprehensive psychiatry, 12(6), 526-536. https://doi.org/10.1016/0010-440X(71)90035-6

Capuano, A. (2016). 'class' discrimination and its relevance to the Australian context. University of New South Wales Law Journal, 39(1).

Chen, G. M., \& Chung, J. (1994). The impact of Confucianism on organizational communication. Communication Quarterly, 42(2), 93-105. https://doi.org/10.1080/01463379409369919

Cowen, T. (2017). The Complacent Class: The Self-Defeating Quest for the American Dream. St. Martin's Press.

Ezekiel, M. (1938).The cobweb theorem. The Quarterly Journal of Economics, 52(2), 255-280. https://doi.org/10.2307/1881734

Gandy, O. H. et al. (2016). Coming to terms with chance: Engaging rational discrimination and cumulative 
disadvantage. Routledge.

Glover, D., Pallais, A., \& Pariente, W. (2017). Discrimination as a self-fulfilling prophecy: Evidence from french grocery stores. The Quarterly Journal of Economics, page qjx006. https://doi.org/10.1093/qje/qjx006

Greener, I. (2006). Nick leeson and the collapse of Barings bank: socio-technical networks and the rogue trader. Organization, 13(3), 421-441. https://doi.org/10.1177/1350508406063491

Hassler, J., Mora, R. J., \& Zeira, J. (2007a). Inequality and mobility. Journal of Economic Growth, 12(3), 235-259. https://doi.org/10.1007/s10887-007-9019-x

Hassler, J., Storesletten, K., \& Zilibotti, F. (2007b). Democratic public good provision. Journal of Economic Theory, 133(1), 127-151. https://doi.org/10.1016/j.jet.2005.07.013

Hwang, K. K. (2006). Moral face and social face: Contingent self-esteem in Confucian society. International Journal of Psychology, 41(4), 276-281. https://doi.org/10.1080/00207590544000040

Jones, G, W. (2007). Delayed marriage and very low fertility in pacific Asia. Population and Development Review, 33(3), 453-478. https://doi.org/10.1111/j.1728-4457.2007.00180.x

Jones, G. (2012). Late marriage and low fertility in Singapore: The limits of policy. The Japanese Journal of Population, 10(1), 89-101.

Kunze, F., Boehm, S. A., \& Bruch, H. (2011). Age diversity, age discrimination climate and performance consequences across organizational study. Journal of organizational behavior, 32(2), 264-290. https://doi.org/10.1002/job.698

Leuven, E., Oosterbeek, H., et al. (2011). Overeducation and mismatch in the labor market. Handbook of the Economics of Education, 4, 283-326. https://doi.org/10.1016/B978-0-444-53444-6.00003-1

Liang, M, Y. (2010). Confucianism and the east Asian miracle. American Economic Journal: Macroeconomics, 2(3), 206-234. https://doi.org/10.1257/mac.2.3.206

Marginson, S. (2011). Higher education in east Asia and Singapore: Rise of the Confucian model. Higher education, 61(5), 587-611. https://doi.org/10.1007/s10734-010-9384-9

Maslow, A, H. (1943). A theory of human motivation. Psychological review, 50(4), 370. https://doi.org/10.1037/h0054346

McArdle, J. J., Ferrer, C. E., Hamagami, F., \& Woodcock, R. W. (2002). Comparative longitudinal structural analyses of the growth and decline of multiple intellectual abilities over the life span. Developmental psychology, 38(1), 115. https://doi.org/10.1037/0012-1649.38.1.115

McDaniel, S. A., Wong, L. L., \& Watt, B. (2015). An aging workforce and the future labor market in Canada.

Park, M., \& Catherine, C. C. (2007). Revisiting Confucianism as a conceptual framework for Asian family study. Journal of Family Nursing, 13(3), 293-311. https://doi.org/10.1177/1074840707304400

Pykett, J. (2009). Personalization and de-schooling: Uncommon trajectories in contemporary education policy. Critical Social Policy, 29(3), 374-397. https://doi.org/10.1177/0261018309105176

Retherford, R. D., Ogawa, N., \& Matsukura, R. (2001). Late marriage and less marriage in japan. Population and development review, 27(1), 65-102. https://doi.org/10.1111/j.1728-4457.2001.00065.x

Saunders, P., Bradbury, B., Wong, M. et al. (2016). The growing gap between rich and poor in Australia. Australian Journal of Labor Economics, 19(1), 15.

Shore, L. M., \& Goldberg, C, B. (2005). Age discrimination in the workplace. Discrimination at work: The psychological and organizational bases, 203-225.

Solon, G. A. (2004). Model of intergenerational mobility variation over time and place. Generational income mobility in North America and Europe, pages 38-47. https://doi.org/10.1017/CBO9780511492549.003

Sunde, U. (2005). Human capital formation, life expectancy, and the process of development. The American Economic Review, 95(5), 1653-1672. https://doi.org/10.1257/000282805775014380

Weber, M. (2002). The Protestant ethic and the spirit of capitalism and other writings. Penguin.

\section{Copyrights}

Copyright for this article is retained by the author(s), with first publication rights granted to the journal.

This is an open-access article distributed under the terms and conditions of the Creative Commons Attribution license which permits unrestricted use, distribution, and reproduction in any medium, provided the original work is properly cited. 